PROCEEDINGS OF THE

AMERICAN MATHEMATICAL SOCIETY

Volume 138, Number 2, February 2010, Pages 533-544

S 0002-9939(09)10120-X

Article electronically published on October 7, 2009

\title{
EQUIVALENCES INDUCED BY INFINITELY GENERATED TILTING MODULES
}

\author{
SILVANA BAZZONI \\ (Communicated by Birge Huisgen-Zimmermann)
}

\begin{abstract}
We generalize Brenner and Butler's Theorem as well as Happel's Theorem on the equivalences induced by a finitely generated tilting module over Artin algebras, to the case of an infinitely generated tilting module over an arbitrary associative ring establishing the equivalences induced between subcategories of module categories and also at the level of derived categories.
\end{abstract}

\section{INTRODUCTION}

Tilting theory started in the context of finitely generated modules over Artin algebras and was further generalized over arbitrary associative rings with unit and to infinitely generated modules (see [6], 8], 9, 1]).

One of the most important features in classical tilting theory is the famous Brenner and Butler's Theorem [5] establishing two equivalences between suitable categories of finitely generated modules.

A finitely generated tilting module $T$ over an Artin algebra $\Lambda$ gives rise to a torsion pair $(\mathcal{T}, \mathcal{F})$, where $\mathcal{T}$ is the class of modules generated by $T$. If $D$ denotes the standard duality and $\Gamma$ is the endomorphism ring of $T$, then $D(T)$ is a cotilting $\Gamma$-module with an associated torsion pair $(\mathcal{X}, \mathcal{Y})$, where $\mathcal{Y}$ is the class of modules cogenerated by $D(T)$. The Brenner and Butler Theorem states that the functor $\operatorname{Hom}_{\Lambda}(T,-)$ induces an equivalence between the categories $\mathcal{T}$ and $\mathcal{Y}$ with inverse the functor $-\otimes_{\Gamma} T$, and the functor $\operatorname{Ext}_{\Lambda}^{1}(T,-)$ induces an equivalence between $\mathcal{F}$ and $\mathcal{X}$ with inverse the functor $\operatorname{Tor}_{1}^{\Gamma}(-, T)$. (See [16] and [17].)

Moreover, $\mathcal{T}$ is the kernel of the functor $\operatorname{Ext}_{\Lambda}^{1}(T,-), \mathcal{Y}$ is the kernel of $\operatorname{Tor}_{1}^{\Gamma}(-, T)$, $\mathcal{F}$ is the kernel of $\operatorname{Hom}_{\Lambda}(T,-)$ and $\mathcal{X}$ is the kernel of $-\otimes_{\Gamma} T$.

Later on, Happel 15 observed that the natural context in which to interpret the above equivalences is that of derived categories. He proved that the total right derived functor of the functor $\operatorname{Hom}_{\Lambda}(T,-)$ induces a derived equivalence between the bounded derived category of finitely generated $\Lambda$-modules and the bounded derived category of finitely generated $\Gamma$-modules.

Received by the editors October 8, 2008, and, in revised form, July 2, 2009

2000 Mathematics Subject Classification. Primary 16D90, 16E30, 18E30; Secondary 16S90, $16 \mathrm{G} 10$.

Key words and phrases. Tilting modules, equivalences and derived equivalences.

Supported by MIUR, PRIN 2005, project "Perspectives in the theory of rings, Hopf algebras and categories of modules" and by Università di Padova (Progetto di Ateneo CPDA071244/07 "Algebras and cluster categories").

(C)2009 American Mathematical Society Reverts to public domain 28 years from publication 
Colby and Fuller 6] proved a "Tilting Theorem" for finitely presented tilting modules over an arbitrary associative ring, generalizing Brenner and Butler's Theorem, and Colpi [7 extended the Tilting Theorem to the wider context of Grothendieck categories.

The first instance of a generalization of Brenner and Butler's Theorem to infinitely generated tilting modules appears in two papers by Facchini [11, [12] where he studied the equivalences induced by the tilting module $\partial$, a divisible module introduced by Fuchs [13] over commutative domains. The theorems proved by Facchini provide a link between the Brenner and Butler tilting equivalences and the equivalences established by Harrison and Matlis between subcategories of modules over a commutative domain $R$.

If $Q$ is the quotient field of a commutative domain $R$ and $K$ is the module $Q / R$, then Harrison and Matlis' Theorem states that the functor $\operatorname{Hom}_{R}(K,-)$ induces an equivalence between the category of $h$-divisible torsion modules and the category of torsion-free cotorsion modules. Moreover, the functor $\operatorname{Ext}^{1}(K,-)$ gives an equivalence between the category of $h$-reduced torsion $R$-modules and the category of special cotorsion modules. Thus the similarity with tilting equivalences was evident, and the papers by Facchini showed the advantage of working with a tilting module, namely the module $\partial$ rather than the module $K$, even though the formal definition of an infinitely generated tilting module was not yet available.

In this paper we generalize both Brenner and Butler's Theorem and Facchini's results to the case of an arbitrary (infinitely generated) tilting module over an associative ring $R$. If Mod- $R$ is the category of all right $R$-modules and $T \in \operatorname{Mod}-R$ is a tilting module, then $T$ induces a torsion pair $(\mathcal{T}, \mathcal{F})$ in $\operatorname{Mod}-R$, where $\mathcal{T}$ is the class of modules generated by $T$. If $S$ is the endomorphism ring of $T$, we prove that the dual $T^{d}$ of $T$ with respect to an injective cogenerator of Mod- $R$ is a partial cotilting right $S$-module inducing a torsion pair $\left(\mathcal{T}_{T^{d}}, \mathcal{F}_{T^{d}}\right)$ in Mod- $S$.

By Theorem 4.5, we prove that the functor $\operatorname{Hom}_{R}(T,-)$ induces an equivalence between the category $\mathcal{T}$ and the intersection of $\mathcal{F}_{T^{d}}$ with a suitable subcategory $\mathcal{M}$ of Mod- $S$, namely the double perpendicular category of the module $T^{d}$ (see the definition in Section (4). Secondly, the functor $\operatorname{Ext}_{R}^{1}(T,-)$ induces an equivalence between $\mathcal{F}$ and the intersection of $\mathcal{T}_{T^{d}}$ with the subcategory $\mathcal{M}$. Moreover, the inverses of these equivalences are given by the functors $-\otimes_{S} T$ and $\operatorname{Tor}_{1}^{S}(-, T)$.

The subcategories of Mod- $S$ equivalent to $\mathcal{T}$ and $\mathcal{F}$ in the above equivalences cannot be interpreted as Gabriel quotients of Mod- $S$, since there are no Serre subcategories arising in the process. Thus again, as in the case of finitely generated tilting modules, the situation can be better illustrated in the context of derived categories, where the equivalences involved can be formulated in a concise and more expressive way. In fact, if $\mathcal{D}(R)$ and $\mathcal{D}(S)$ are the (unbounded) derived categories of the categories Mod- $R$ and Mod- $S$, respectively, we prove that the total right derived functor of the functor $\operatorname{Hom}_{R}(T,-)$, that is, the functor $\mathbb{R H o m}(T,-)$, induces an equivalence between $\mathcal{D}(R)$ and the quotient category of $\mathcal{D}(S)$ modulo

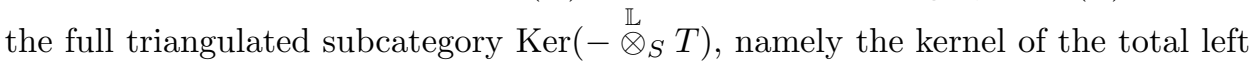
derived functor of the functor $-\otimes_{S} T$.

\section{Preliminaries}

In what follows all rings are associative with unit. We recall some definitions and results. 
For a ring $R, \operatorname{Mod}-R$ ( $R$-Mod) will denote the category of all right (left) $R$ modules. For an $R$-module $M$ we denote by p.d. $M$ and i.d. $M$ the projective and injective dimensions of $M$, respectively.

If $\lambda$ is a cardinal, $M^{(\lambda)}$ and $M^{\lambda}$ will denote the direct sum and the direct product of $\lambda$ copies of $M$, respectively.

Let $\mathcal{C} \subseteq$ Mod- $R$. Define

$$
\begin{aligned}
& \mathcal{C}^{\perp}=\left\{X \in \operatorname{Mod}-R \mid \operatorname{Ext}_{R}^{i}(C, X)=0 \text { for all } C \in \mathcal{C}, \text { for all } i \geq 1\right\} \\
& { }^{\perp} \mathcal{C}=\left\{X \in \operatorname{Mod}-R \mid \operatorname{Ext}_{R}^{i}(X, C)=0 \text { for all } C \in \mathcal{C}, \text { for all } i \geq 1\right\}
\end{aligned}
$$

Definition 2.1 ([8, [1]). An $R$-module $T$ is 1-tilting provided

(T1) p.d.T $\leq 1$,

(T2) $\operatorname{Ext}_{R}^{i}\left(T, T^{(\lambda)}\right)=0$ for each $i \geq 1$ and every cardinal $\lambda$, and

(T3) there exists an exact sequence

$$
0 \rightarrow R \rightarrow T_{0} \rightarrow T_{1} \rightarrow 0
$$

such that $T_{i} \in$ Add $T$ for each $0 \leq i \leq 1$.

Here, Add $T$ denotes the class of all direct summands of arbitrary direct sums of copies of $T$.

If $T$ is a 1-tilting module, $T^{\perp}$ is called a 1-tilting class.

Definition 2.2 ([8]). An $R$-module $T$ is 1-partial tilting if $T$ satisfies (T1), (T2) and $T^{\perp}$ is closed under direct sums.

We have also dual definitions.

Definition 2.3 ([9, 1]). A module $C$ is 1-cotilting provided

(C1) i.d. $C \leq 1$,

(C2) $\operatorname{Ext}_{R}^{i}\left(C^{\lambda}, C\right)=0$ for each $i \geq 1$ and every cardinal $\lambda$, and

(C3) there exists an exact sequence

$$
0 \rightarrow C_{1} \rightarrow C_{0} \rightarrow W \rightarrow 0
$$

such that $C_{i} \in \operatorname{Prod} C$ for each $0 \leq i \leq 1$ and $W$ is an injective $R$-cogenerator.

Here, Prod $C$ denotes the class of all direct summands of arbitrary direct products of copies of $C$.

If $C$ is a 1-cotilting module, ${ }^{\perp} C$ is called a 1 -cotilting class.

Definition $2.4(9])$. An $R$-module $C$ is 1-partial cotilting if $C$ satisfies (C1), (C2) and ${ }^{\perp} C$ is closed under direct products.

If $T$ and $U$ are 1-tilting (1-cotilting) modules, then $T$ is equivalent to $U$ if $T^{\perp}=$ $U^{\perp}\left({ }^{\perp} T={ }^{\perp} U\right)$, which is the case if and only if $\operatorname{Add} T=\operatorname{Add} U(\operatorname{Prod} T=$ Prod $U)$.

We recall some results on infinitely generated 1-tilting and 1-cotilting modules which give a better understanding of their properties.

- By [8, 1.3] a module $T$ is 1-tilting if and only if $T^{\perp}=$ GenT, where GenT is the class of modules generated by $T$.

- By [3] if $T$ is a 1-tilting module, then the tilting class $T^{\perp}$ is of finite type; that is, there is a set $\mathcal{S}$ of finitely presented modules of projective dimension at most 1 , such that $\mathcal{S}^{\perp}=T^{\perp}$.

- By [2] 1-cotilting modules are pure injective. 
- As a consequence of the above results, we have that every 1-tilting right $R$ module $T$ induces a torsion pair $(\mathcal{T}, \mathcal{F})$ in $\operatorname{Mod}-R$, where $\mathcal{T}=\operatorname{Gen} T=T^{\perp}$ and $\mathcal{F}=\operatorname{Ker}\left(\operatorname{Hom}_{R}(T,-)\right)$.

Every 1-cotilting right $R$-module $C$ induces a torsion pair $(\mathcal{T}, \mathcal{F})$ in $\operatorname{Mod}-R$ where $\mathcal{F}=\operatorname{Cogen} C={ }^{\perp} C$ and $\mathcal{T}=\operatorname{Ker}\left(\operatorname{Hom}_{R}(-, C)\right)$.

\section{INFINITELY GENERATED 1-TILTING MODULES}

In this section we adapt the results proved by Facchini in [11 and [12] for the case of the tilting module $\partial$ defined over a commutative domain to the case of a tilting module over an arbitrary associative ring.

First of all we have to make a suitable choice of a representative in the equivalence class of a 1-tilting module.

Proposition 3.1. Let $R$ be a ring and let $T_{R}$ be a 1-tilting module. Up to equivalence we can assume that $T$ fits in an exact sequence of the form

$$
0 \rightarrow R \rightarrow T \rightarrow T_{1} \rightarrow 0,
$$

where $T_{1}$ is a direct summand of $T$.

Proof. From condition (T3) in the definition of tilting modules, we have an exact sequence

$$
0 \rightarrow R \stackrel{\iota}{\rightarrow} T_{0} \rightarrow T_{1} \rightarrow 0
$$

where $T_{0}, T_{1} \in$ Add $T$. Consider the module $T^{\prime}=T_{0} \oplus\left(T_{1}\right)^{(\omega)}$ and let $j: T_{0} \rightarrow T^{\prime}$ be the natural embedding of $T_{0}$ in $T^{\prime}$. Then we have an exact sequence

$$
0 \rightarrow R \stackrel{\text { jol }}{\rightarrow} T^{\prime} \rightarrow T_{1} \oplus\left(T_{1}\right)^{(\omega)} \rightarrow 0
$$

where $T_{1} \oplus\left(T_{1}\right)^{(\omega)} \cong\left(T_{1}\right)^{(\omega)}$ is isomorphic to a direct summand of $T^{\prime}$. Thus we also have an exact sequence

$$
0 \rightarrow R \rightarrow T^{\prime} \rightarrow T_{1}^{\prime} \rightarrow 0
$$

with $T_{1}^{\prime}$ a direct summand of $T^{\prime}$. Now $T^{\prime}$ is a 1-tilting module. In fact, $T^{\prime}$ satisfies conditions (T1) and (T2) since $T^{\prime} \in$ Add $T$; it satisfies also (T3), by the above sequence. Moreover, $T^{\prime}$ and $T$ are equivalent, since $T^{\perp} \subseteq T^{\perp}$ and $T^{\prime \perp}=\mathrm{Gen} T^{\prime} \subseteq$ Gen $T=T^{\perp}$.

Definition 3.2. We say that $T_{R}$ is a good 1-tilting module if it is a 1-tilting module such that the short exact sequence of condition (T3) has the form

$$
\text { (a) } \quad 0 \rightarrow R \stackrel{\mu}{\rightarrow} T \rightarrow T_{1} \rightarrow 0,
$$

where $T_{1}$ is a direct summand of $T$.

We denote by $S$ the endomorphism ring of $T$. As in [11 we fix the following notation:

(1) $\mu\left(1_{R}\right)=w \in T$.

(2) Let $\phi \in S$ be the composition $T \rightarrow T_{1} \rightarrow T$; then $\phi$ is an endomorphism of $T$ such that $\operatorname{Ker} \phi=w R$ and $\phi(T)$ is a direct summand of $T$.

(3) $e$ is a fixed idempotent endomorphism of $T$ such that $e(T)=\phi(T)$.

Lemma 3.3. Let $T_{R}$ be a good 1-tilting module. There is a short exact sequence of left $S$-modules

$$
\text { (b) } \quad 0 \rightarrow I \rightarrow S \rightarrow T \rightarrow 0
$$

such that 
(1) I is the left ideal $\{f \in S \mid f(w)=0\}$ and also $I=S \phi$;

(2) I is isomorphic to $\mathrm{Se}$;

(3) $\operatorname{End}_{S}(T) \cong R$;

(4) ${ }_{S} T$ is a cyclically presented partial 1-tilting $S$-module.

Proof. (1) and (2) follow by applying the functor $\operatorname{Hom}_{R}(-, T)$ to the exact sequence (a).

So ${ }_{S} T$ is cyclically presented; (3) and (4) follow by [8, Lemma 2.15].

Proposition 3.4 ([8], [12]). Let $T_{R}$ be a good 1-tilting module. The following hold:

(1) The natural homomorphism (the counit of the adjunction)

$$
\phi: \operatorname{Hom}_{R}(T, M) \otimes_{S} T \rightarrow M
$$

is an isomorphism if and only if $M$ is in the tilting class $T^{\perp}$.

(2) $\operatorname{Tor}_{1}^{S}\left(\operatorname{Hom}_{R}(T, M), T\right)=0$, for every right $R$-module $M$.

Proof. (1) is proved in [8, Corollary 2.18].

(2) The proof is the same as in [11, Proposition 4.2], but we repeat the argument because our context is different. Let $N$ be a right $S$-module; applying the functor $N \otimes_{S}$ - to the exact sequence (b), we get that $\operatorname{Tor}_{1}^{S}(N, T)$ is the kernel of the map $N \otimes_{S} I \rightarrow N$. Since $I=S \phi \cong S e$ we have that $\operatorname{Tor}_{1}^{S}(N, T)$ is isomorphic to the kernel of the abelian group morphism $N e \rightarrow N$ defined by $x e \mapsto x \phi$, for every $x \in N$; hence $\operatorname{Tor}_{1}^{S}(N, T)$ is isomorphic to $\{x \in N \mid x \phi=0\} e$.

So we need to show that, for every right $R$-module $M$, if $Y=\left\{g \in \operatorname{Hom}_{R}(T, M) \mid\right.$ $g \phi=0\}$, then $Y e=0$. Now $g \phi=0$ if and only if Ker $g \supseteq \phi(T)=e T$ if and only if $g e=0$.

\section{Equivalences Between SUbClasses of MODUles}

For every right $R$-module $M$ we denote by $M^{d}$ the dual of $M$ with respect to an injective cogenerator $W$ of Mod- $R$, that is, $M^{d}=\operatorname{Hom}_{R}(M, W)$.

Proposition 4.1. Let $T_{R}$ be a good 1-tilting module. The right $S$-module $T^{d}$ satisfies the following properties:

(1) $\left[\operatorname{Tor}_{i}^{S}(-, T)\right]^{d} \cong \operatorname{Ext}_{S}^{i}\left(-, T^{d}\right)$. In particular, i.d. $\left(T^{d}\right)_{S} \leq 1$.

(2) $\operatorname{Tor}_{i}^{S}\left(T^{d}, T\right) \cong\left[\operatorname{Ext}_{S}^{i}(T, T)\right]^{d}=0$.

(3) $T^{d}$ is a partial 1-cotilting right $S$-module.

Proof. (1) This follows by a well-known Ext-Tor relation and i.d. $\left(T^{d}\right)_{S} \leq 1$, since p.d. $S T \leq 1$, so $\operatorname{Tor}_{i}^{S}(-, T)=0$, for every $i \geq 2$.

(2) ${ }_{S} T$ is a finitely presented left $S$-module and p.d. ${ }_{S} T \leq 1$; hence $\operatorname{Tor}_{i}^{S}\left(T^{d}, T\right) \cong$ $\left[\operatorname{Ext}_{S}^{i}(T, T)\right]^{d}$ and $\operatorname{Ext}_{S}^{i}(T, T)=0$, for every $i \geq 1$, since ${ }_{S} T$ is a partial 1-tilting module, by Lemma 3.3 .

(3) By (1) i.d. $\left(T^{d}\right)_{S} \leq 1$. Let $\left\{N_{i}\right\}_{i}$ be a family of right $S$-modules such that $\operatorname{Ext}_{S}^{1}\left(N_{i}, T^{d}\right)=0$, for every $i$. By $(1)$ we have $\operatorname{Tor}_{1}^{S}\left(N_{i}, T\right)=0$. We show that $\operatorname{Ext}_{S}^{1}\left(\prod_{i} N_{i}, T^{d}\right)=0$. By (1) again, we have $\operatorname{Ext}_{S}^{1}\left(\prod_{i} N_{i}, T^{d}\right) \cong\left[\operatorname{Tor}_{1}^{S}\left(\prod_{i} N_{i}, T\right)\right]^{d}$. Since ${ }_{S} T$ is finitely presented and p.d. ${ }_{S} T \leq 1$, $\operatorname{Tor}_{1}^{S}(-, T)$ commutes with direct products; hence $\operatorname{Ext}_{S}^{1}\left(\prod_{i} N_{i}, T^{d}\right) \cong\left[\prod_{i}\left(\operatorname{Tor}_{1}^{S}\left(N_{i}, T\right)\right]^{d}\right.$. But, as noted above, $\operatorname{Tor}_{1}^{S}\left(N_{i}, T\right)=0$. Thus, ${ }^{\perp} T^{d}$ is closed under direct products. To conclude that $T^{d}$ is a partial 1-cotilting module, it is enough to check that $\operatorname{Ext}_{S}^{1}\left(T^{d}, T^{d}\right)=0$. Now, $\operatorname{Ext}_{S}^{1}\left(T^{d}, T^{d}\right) \cong\left[\operatorname{Tor}_{1}^{S}\left(T^{d}, T\right)\right]^{d}$ and $\operatorname{Tor}_{1}^{S}\left(T^{d}, T\right)=0$ by $(2)$. 
Recall that if $M$ is an $R$-module over a ring $R$, the preradical $\operatorname{Rej}_{M}$ is the subfunctor of the identity functor defined by $\operatorname{Rej}_{M}(X)=\bigcap \operatorname{Ker}\left\{f \mid f \in \operatorname{Hom}_{R}(X, M)\right\}$, for every $R$-module $X$. Rej ${ }_{M}$ is always a radical and if it is also idempotent, then it is a torsion radical (see 21]). In this case the associated torsion class consists of the modules $X$ such that $\operatorname{Hom}_{R}(X, M)=0$ and the torsion-free class is Cogen $M$.

Proposition 4.2. In the same notation as in Proposition 4.1, the partial 1-cotilting $S$-module $T^{d}$ satisfies the following conditions:

(1) $T_{S}^{d}$ is a direct summand of a 1-cotilting right $S$-module $C$ such that ${ }^{\perp} C=$ ${ }^{\perp} T^{d}$.

(2) The preradical $\operatorname{Rej}_{T^{d}}$ is an idempotent radical inducing a torsion pair $\left(\mathcal{T}_{T^{d}}\right.$, $\mathcal{F}_{T^{d}}$ ) in Mod-S, where $\mathcal{F}_{T^{d}}=$ Cogen $T^{d} \subseteq{ }^{\perp} T^{d}$.

Proof. (1) See [10, Theorem 2.11].

(2) This follows by [10, Lemma 2.6] and Cogen $T^{d} \subseteq{ }^{\perp} T^{d}$, since $T^{d}$ is a partial 1-cotilting module, by Proposition 4.1.

We now define the subcategories of Mod- $S$ which will play a crucial role in establishing the equivalences which will be proved by Theorem 4.5 .

First, we recall a notion of perpendicular categories. If $\mathcal{C}$ is a category of $R$ modules we define the right perpendicular category $\mathcal{C}_{\perp}$ as

$$
\mathcal{C}_{\perp}=\left\{M \mid \operatorname{Hom}_{R}(\mathcal{C}, M)=\operatorname{Ext}_{R}^{1}(\mathcal{C}, M)=0\right\}
$$

and analogously the left perpendicular category as

$$
{ }_{\perp} \mathcal{C}=\left\{M \mid \operatorname{Hom}_{R}(M, \mathcal{C})=\operatorname{Ext}_{R}^{1}(M, \mathcal{C})=0\right\} .
$$

We will also use the following definitions.

Definition 4.3. Let $\mathcal{C}$ be a subcategory of an abelian category $\mathcal{A}$.

(1) $\mathcal{C}$ has the 2 out of 3 property if for every short exact sequence

$$
0 \rightarrow L \rightarrow M \rightarrow N \rightarrow 0
$$

in $\mathcal{A}$ with two terms in $\mathcal{C}$, then the third term is also in $\mathcal{C}$.

(2) $\mathcal{C}$ is a Serre subcategory if for every short exact sequence

$$
0 \rightarrow L \rightarrow M \rightarrow N \rightarrow 0
$$

in $\mathcal{A}, M$ is in $\mathcal{C}$ if and only if $L$ and $N$ are in $\mathcal{C}$.

Proposition 4.4. Let

$$
\mathcal{E}=\left\{N \in \operatorname{Mod}-S \mid N \otimes_{S} T=\operatorname{Tor}_{1}^{S}(N, T)=0\right\} .
$$

The following hold:

(1) $\mathcal{E}=\left\{N \in \operatorname{Mod}-S \mid \operatorname{Ext}_{S}^{1}\left(N, T^{d}\right)=\operatorname{Hom}_{S}\left(N, T^{d}\right)=0\right\}$, that is, $\mathcal{E}={ }_{\perp}\left\{T^{d}\right\}$.

(2) $\mathcal{E}$ is closed under direct sums, direct summands and has the 2 out of 3 property.

(3) $\mathcal{E}={ }^{\perp} T^{d} \cap \mathcal{T}_{T^{d}}$.

Proof. (1) The equality follows by the usual homological formulas.

(2) This follows by a direct check.

(3) The equality follows by (1). 
Consider now the right perpendicular category $\mathcal{M}$ of $\mathcal{E}$, that is,

$$
\mathcal{M}=\mathcal{E}_{\perp}=\left\{M \in \operatorname{Mod}-S \mid \operatorname{Hom}_{S}(\mathcal{E}, M)=0=\operatorname{Ext}_{S}^{1}(\mathcal{E}, M)\right\} .
$$

The next theorem, inspired by Facchini's Theorems, is the generalization of the equivalences proved by Brenner and Butler 5 in the case of a classical 1-tilting module (that is finitely generated) over Artin algebras.

Theorem 4.5 (11, 12). Let $R$ be a ring, $T_{R}$ a good 1-tilting module and let $(\mathcal{T}, \mathcal{F})$ be the tilting torsion pair associated to $T$. Let $S=\operatorname{End}_{R}(T)$. The following hold:

(1) There is an equivalence

$$
\operatorname{Mod}-R \supseteq \mathcal{T}^{\operatorname{Hom}_{R}(T,-)} \mathcal{Y} \subseteq \operatorname{Mod}-S,
$$

where $\mathcal{Y}=\mathcal{F}_{T^{d}} \cap \mathcal{M}$ with inverse $-\otimes_{S} T$.

(2) There is an equivalence

$$
\operatorname{Mod}-R \supseteq \mathcal{F} \stackrel{\operatorname{Ext}_{R}^{1}(T,-)}{\longrightarrow} \mathcal{X} \subseteq \operatorname{Mod}-S,
$$

where $\mathcal{X}=\mathcal{T}_{T^{d}} \cap \mathcal{M}$ with inverse $\operatorname{Tor}_{1}^{S}(-, T)$.

Proof. The proof of the two equivalences is essentially the same as [11] and [12], with the suitable translation of the terminology.

In those papers the modules named $I$-divisible are the modules in $\mathcal{T}_{T^{d}}$, that is, the right $S$-modules $N$ such that $\operatorname{Hom}_{S}\left(N, T^{d}\right)=0$ or equivalently, $N \otimes_{S} T=0$. The modules called $I$-reduced are the modules in $\mathcal{F}_{T^{d}}$. Moreover, the modules in the class $\mathcal{E}$ are called $I$-divisible and $I$-torsion-free.

(1) This is proved by the same arguments as in [1], once it is observed that the modules named $I$-cotorsion in that paper are the modules in the class $\mathcal{F}_{T^{d}} \cap \mathcal{M}$.

First one shows as in [11, Theorem 7.1] that for every $M \in \operatorname{Mod}-R, \operatorname{Hom}_{R}(T, M)$ $\in \mathcal{F}_{T^{d}} \cap \mathcal{M}$. Then one uses that, by Proposition 3.4 (1), $\phi: \operatorname{Hom}_{R}(T, M) \otimes_{S} T \rightarrow M$ is an isomorphism if and only if $M$ is in the tilting torsion class $\mathcal{T}$. Finally one verifies that $\eta: N \rightarrow \operatorname{Hom}_{R}\left(T, N \otimes_{S} T\right)$ is an isomorphism if and only if $N$ is a right $S$-module in the class $\mathcal{F}_{T^{d}} \cap \mathcal{M}$. This is obtained by following the proofs of [11. Theorem 7.2, 7.3].

(2) This is proved as in [12] by noticing that there, slightly differently from the definitions in [11, the modules named $I$-cotorsion are the modules in the class $\mathcal{M}$.

First, as in [12, Lemma 1], one proves that $\operatorname{Ext}_{R}^{1}(T, M)$ is in the class $\mathcal{T}_{T^{d}} \cap \mathcal{M}$, for every right $R$-module $M$. Secondly, one shows that if $M$ is in the torsionfree class $\mathcal{F}$, then the natural homomorphism $\xi: \operatorname{Tor}_{1}^{S}\left(\operatorname{Ext}_{R}^{1}(T, M), T\right) \rightarrow M$ is an isomorphism (see [12, Lemma 1]).

Then, one proves that $\operatorname{Tor}_{1}^{S}(N, T) \in \mathcal{F}$, for every $N \in \mathcal{T}_{T^{d}} \cap \mathcal{M}$, and that the natural homomorphism $\theta: N \rightarrow \operatorname{Ext}_{R}^{1}\left(T, \operatorname{Tor}_{1}^{S}(N, T)\right)$ is an isomorphism if and only if $N \in \mathcal{T}_{T^{d}} \cap \mathcal{M}$ (see [12, Lemma 2]).

Remark 1. If $T$ is a finitely presented 1-tilting module, then the dual module $T^{d}$ is a 1-cotilting module over the endomorphism ring of $T$. Hence, in this case, the category $\mathcal{E}={ }_{\perp} T^{d}$ is zero, so $\mathcal{M}$ coincides with $\operatorname{Mod}-S$ and $(\mathcal{X}, \mathcal{Y})$ is the cotilting torsion pair associated to the 1-cotilting module $T^{d}$. Thus, we recover both Brenner and Butler's Theorem for the case of Artin algebras and the ColbyFuller Tilting theorem over an arbitrary ring. So, Theorem 4.5 can be viewed 
as the generalization to the case of infinitely generated 1-tilting modules of the Brenner-Butler and Colby-Fuller Theorems.

The categories $\operatorname{Ker}\left(-\otimes_{S} T\right)$ and $\operatorname{Ker}\left(\operatorname{Tor}_{1}^{S}(-, T)\right)$ are not Serre subcategories of Mod- $S$ in general. Thus, we cannot perform the corresponding quotient categories in Gabriel's sense. However, we can localize the category Mod-S at a suitable multiplicative system, as we are going to explain.

In the next proposition we use the terminology as in Gabriel and Zisman's book [14.

Proposition 4.6. Let $T_{R}$ be a good 1-tilting module and let $(\mathcal{T}, \mathcal{F})$ be the associated torsion pair in Mod- $R$. Let $\Sigma$ be the system of morphisms $u \in \operatorname{Mod}-S$ such that $u \otimes_{S} 1_{T}$ is invertible in Mod- $R$. Then the following hold:

(1) $\Sigma$ admits a calculus of left fractions.

(2) There is an equivalence $\rho: \operatorname{Mod}-S\left[\Sigma^{-1}\right] \rightarrow \mathcal{T}$ such that $\rho \circ q=-\otimes_{S} T$, where $q$ : Mod- $S \rightarrow \operatorname{Mod}-S\left[\Sigma^{-1}\right]$ is the canonical localization functor.

(3) There is an equivalence between Mod-S[ $\left[\Sigma^{-1}\right]$ and the category $\mathcal{Y}=\mathcal{F}_{T^{d}} \cap$ $\mathcal{M}$.

Proof. (1) Note that $N \otimes_{S} T \in \mathcal{T}$ for every right $S$-module $N$ and that $\mathcal{T}$ is a full subcategory of Mod- $R$. Hence the functor $H=\operatorname{Hom}_{R}(T,-): \mathcal{T} \rightarrow \operatorname{Mod}-S$ is right adjoint to the functor $G=-\otimes_{S} T: \operatorname{Mod}-S \rightarrow \mathcal{T}$. By Proposition 3.4 (1), the counit adjunction $\phi: G H \rightarrow 1_{\mathcal{T}}$ is invertible, and, by [14, Proposition 1.3], $H$ is a fully faithful functor. Hence $\Sigma$ admits a calculus of left fractions by [14, 2.5 (b)].

(2) This follows by Proposition 3.4 (1) and by [14, Proposition 1.3].

(3) Combine (2) with Theorem 4.5(1).

Remark 2. We couldn't get an analogous result for the pair of functors $\operatorname{Ext}_{R}^{1}(T,-)$ and $\operatorname{Tor}_{1}^{S}(-, T)$ because they are not an adjoint pair in general.

Moreover, we don't know whether the category of fractions Mod- $S\left[\Sigma^{-1}\right]$, considered in Proposition 4.6, is the quotient of Mod- $S$ modulo a suitable subcategory.

The above remark indicates that a better understanding of the whole situation can be obtained in the setting of derived categories.

\section{Derived equivalence}

Before stating the main result of this section we recall some notions and facts about derived categories which will be used later on.

Let $\mathcal{D}(R)$ and $\mathcal{D}(S)$ be the unbounded derived categories of Mod- $R$ and Mod- $S$, respectively. The following hold:

- (Bökstedt and Neeman [4] or Spaltenstein [20]) For every complex $M \cdot \in$ $\mathcal{D}(R)$ there is a quasi isomorphism $M^{\cdot} \rightarrow I^{\prime}$, where $I^{*}$ is a complex with injective terms. $I$ is also denoted by $\underline{\mathbf{i}} M$ and called a $K$-injective or fibrant resolution of $M \cdot$.

Symmetrically, for every complex $M \cdot \in \mathcal{D}(R)$ there is a quasi isomorphism $P^{\cdot} \rightarrow M$, where $P^{\cdot}$ is a complex with projective terms. $P^{\cdot}$ is also denoted by $\underline{\mathbf{p}} M$ and called a $K$-projective or cofibrant resolution of $M$.

- (19, Theorem 3.2 (b)] and Bökstedt and Neeman [4) Every additive functor $F$ defined on the module category Mod- $R$ admits a total right derived functor $\mathbb{R} F$ and a total left derived functor $\mathbb{L} F$ defined on $\mathcal{D}(R)$. 
Moreover, if $M^{\cdot}$ is a complex in $\mathcal{D}(R)$, then $\mathbb{R} F\left(M^{*}\right)=F\left(\underline{\mathbf{i}} M^{\cdot}\right)$ and $\mathbb{L} F\left(M^{*}\right)=F\left(\mathbf{p} M^{*}\right)$. (We also denote by $F$ the functor induced on the homotopy category.)

- (19, Theorem 3.2 (c)]) If $T$ is an $S$-R-bimodule, then the adjoint pair $(G, H)$ of functors given by

$$
H=\operatorname{Hom}_{R}(T,-): \operatorname{Mod}-R \rightleftarrows \operatorname{Mod}-S: G=-\otimes_{S} T
$$

induces an adjoint pair of total derived functors

$$
\mathbb{R} H=\mathbb{R} \operatorname{Hom}_{R}(T,-): \mathcal{D}(R) \rightleftarrows \mathcal{D}(S): \mathbb{L} G=-\stackrel{\mathbb{L}}{\otimes_{S}} T .
$$

Theorem 5.1. Let $T_{R}$ be a good 1-tilting module with endomorphism ring $S$. The following hold:

(1) The counit adjunction morphism

$$
\eta: \mathbb{L} G \circ \mathbb{R} H \rightarrow I d_{\mathcal{D}(R)}
$$

is invertible.

(2) The functor $\mathbb{R} H: \mathcal{D}(R) \rightarrow \mathcal{D}(S)$ is fully faithful.

(3) There is a triangle equivalence $\Theta: \mathcal{D}(S)\left[\Sigma^{-1}\right] \rightarrow \mathcal{D}(R)$ such that $\mathbb{L} G=\Theta \circ q$, where $q$ is the canonical quotient functor $q: \mathcal{D}(S) \rightarrow \mathcal{D}(S)\left[\Sigma^{-1}\right]$.

(4) If $\Sigma$ is the system of morphisms $u \in \mathcal{D}(S)$ such that $\mathbb{L} G u$ is invertible in $\mathcal{D}(R)$, then $\Sigma$ admits a calculus of left fractions and the category $\mathcal{D}(S)\left[\Sigma^{-1}\right]$ coincides with the quotient category $\mathcal{D}(S)$ modulo the full triangulated subcategory $\operatorname{Ker}(\mathbb{L} G)$ of the objects annihilated by the functor $\mathbb{L} G$.

We first prove condition (1) of Theorem 5.1 by a lemma.

Lemma 5.2. In the assumptions of Theorem 5.1, the counit adjunction morphism

$$
\eta: \mathbb{L} G \circ \mathbb{R} H \rightarrow I d_{\mathcal{D}(R)}
$$

is invertible.

Proof. Let $M$. be a complex in $\mathcal{D}(R)$ and consider a $K$-injective resolution $\underline{\mathbf{i}} M$ - of $M$. We have:

$$
\mathbb{R} H\left(M^{\cdot}\right)=\mathbb{R} \operatorname{Hom}_{R}(T,-)\left(M^{*}\right)=H\left(\underline{\mathbf{i}} M^{\cdot}\right) .
$$

Let $C^{\cdot}=H\left(\mathbf{i} M^{\cdot}\right) . C \cdot$ is a complex of right $S$-modules and

$$
\mathbb{L} G\left(C^{\cdot}\right)=\mathbb{L}\left(-\otimes_{S} T\right)\left(C^{\cdot}\right)=G\left(\underline{\mathbf{p}} C^{\cdot}\right),
$$

where $\mathbf{p} C$ is a $K$-projective resolution of $C$ as a complex in $\mathcal{D}(S)$.

Consider the complex $T^{*}: 0 \rightarrow{ }_{S} T \rightarrow 0$ concentrated in degree 0 . A $K$-projective resolution $\mathbf{p} T^{\cdot}$ of $T$ in $\mathcal{D}(S)$ is the complex $0 \rightarrow I \stackrel{\delta}{\rightarrow} S \rightarrow 0$ (from the exact sequence $(\bar{b})$ in Lemma 3.3$)$.

From the quasi isomorphism $\underline{\mathbf{p}} T^{\cdot} \rightarrow T^{\cdot}$ and $\underline{\mathbf{p}} C^{\cdot} \rightarrow C^{\cdot}$ we get the chain of quasi isomorphisms:

$$
G\left(\underline{\mathbf{p}} C^{\cdot}\right)=\underline{\mathbf{p}} C^{\cdot} \otimes_{S} T \leftarrow \underline{\mathbf{p}} C^{\cdot} \otimes_{S} \underline{\mathbf{p}} T^{\cdot} \rightarrow C \cdot \otimes_{S} \underline{\mathbf{p}} T^{*} .
$$

Thus, $\mathbb{L} G\left(C^{\cdot}\right)=C^{\cdot} \otimes_{S} \underline{\mathbf{p}} T^{\cdot}$ and this gives

$$
\mathbb{L} G\left(C^{\cdot}\right)=C \cdot \stackrel{\mathbb{L}}{\otimes_{S}} T=\text { Cone }(1 \otimes \delta) .
$$


From the exact sequence (b) in Lemma 3.3 we obtain the exact sequence of complexes of right $R$-modules:

$$
\operatorname{Tor}_{1}^{S}\left(C^{\cdot}, T\right) \rightarrow C^{\cdot} \otimes_{S} I \rightarrow C^{\cdot} \otimes_{S} S \rightarrow C \cdot \otimes_{S} T \rightarrow 0 .
$$

Now recalling that $C^{\cdot}$ is the complex $\mathbb{R} H\left(M^{*}\right)=H\left(\underline{\mathbf{i}} M^{*}\right)$, Proposition 3.4 (2) yields that the complex $\operatorname{Tor}_{1}^{S}(C, T)$ has zero terms; hence we have the short exact sequence of complexes of right $R$-modules:

$$
\text { (2) } 0 \rightarrow C \cdot \otimes_{S} I \rightarrow C \cdot \otimes_{S} S \rightarrow C \cdot \otimes_{S} T \rightarrow 0,
$$

From (2) we obtain the long exact sequence in cohomology:

$$
\begin{aligned}
& \cdots \rightarrow H^{n+1}\left(C \cdot \otimes_{S} T\right) \rightarrow H^{n}\left(C^{\cdot} \otimes_{S} I\right) \\
& \rightarrow H^{n}\left(C \cdot \otimes_{S} S\right) \rightarrow H^{n}\left(C \cdot \otimes_{S} T\right) \rightarrow \ldots
\end{aligned}
$$

We also have the exact sequence of complexes of right $R$-modules:

$$
0 \rightarrow C \cdot \otimes \rightarrow \text { Cone }(1 \otimes \delta) \rightarrow(C \cdot \otimes I)[1] \rightarrow 0
$$

from which we get the long exact sequence

$$
\begin{gathered}
\cdots \rightarrow H^{n+1}(\text { Cone }(1 \otimes \delta)) \rightarrow H^{n}\left(C \cdot \otimes_{S} I\right) \rightarrow H^{n}\left(C \cdot \otimes_{S} S\right) \\
\rightarrow H^{n}(\text { Cone }(1 \otimes \delta)) \rightarrow C \cdot \otimes_{S} I \rightarrow \ldots
\end{gathered}
$$

Now comparing $\left(^{*}\right)$ with $(* *)$ we conclude that, for every $n \in \mathbb{N}$,

$$
\left.H^{n}\left(C^{\cdot} \otimes_{S} T\right) \cong H^{n}(\operatorname{Cone}(1 \otimes \delta)) \cong H^{n}\left(C^{\cdot \mathbb{L}} \otimes_{S} T\right)\right) .
$$

Hence,

$$
\mathbb{L} G(C \cdot)=C \cdot \stackrel{\mathbb{L}}{\otimes}_{S} T \text { is quasi-isomorphic to } C \cdot \otimes_{S} T .
$$

Letting $I^{\cdot}=\underline{\mathbf{i}} M^{*}$, we have $C^{\cdot}=\operatorname{Hom}_{R}\left(T, I^{\cdot}\right)$ and we have also the commutative diagram:

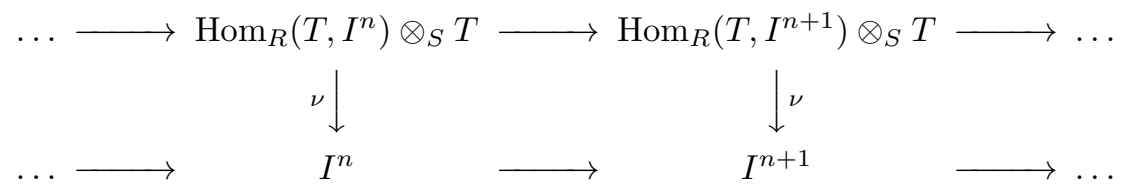

where the vertical maps are canonical isomorphisms by Proposition 3.4 (1), since $I$. is a complex of injective right $R$-modules, hence belonging to the tilting class $T^{\perp}$.

Hence, $\operatorname{Hom}_{R}\left(T, I^{\cdot}\right) \otimes_{S} T$ and $I^{\cdot}$ are canonically isomorphic as complexes of $R$-modules, so we have:

$$
\mathbb{L} G\left(\mathbb{R} H\left(M^{\cdot}\right)\right)=H\left(\underline{\mathbf{i}} M^{\cdot}\right) \stackrel{\mathbb{L}}{\otimes_{S}} T \cong \underline{\mathbf{i}} M \cdot \cong M^{\cdot} .
$$

Proof of Theorem 5.1. Condition (1) is proved by Lemma 5.2, and the equivalence of (1) with the other conditions follows essentially by applying [14, Proposition 1.3].

To complete the proof we add only a few comments.

The equivalence $\Theta: \mathcal{D}(S)\left[\Sigma^{-1}\right] \rightarrow \mathcal{D}(R)$, guaranteed by [14, Proposition 1.3], is a triangle equivalence, since $\mathbb{L} G$ is a triangle functor and $q$ is the canonical localization functor, so that the triangles in $\mathcal{D}(S)\left[\Sigma^{-1}\right]$ are images of triangles in $\mathcal{D}(S)$. 
The functor $\mathbb{L} G=-\mathbb{\mathscr { Q }}_{S} T$ is a triangle functor; hence $\operatorname{Ker}(\mathbb{L} G)$ is a full triangulated subcategory of $\mathcal{D}(S)$. It is well known that the quotient category $\mathcal{D}(S)$ / $\operatorname{Ker}(\mathbb{L} G)$ is the localization of $\mathcal{D}(S)$ at the multiplicative system $\Sigma$ consisting of the morphisms $u \in \mathcal{D}(S)$ such that there exists a triangle

$$
K^{\cdot} \rightarrow M^{\cdot} \stackrel{u}{\rightarrow} N^{*} \rightarrow K^{\cdot}[1],
$$

where $K^{\cdot} \in \operatorname{Ker}(\mathbb{L} G)$ and $M^{*}, N^{\cdot} \in \mathcal{D}(S)$. Thus $\Sigma$ coincides with the system of morphisms $u \in \mathcal{D}(S)$ such that $\mathbb{L} G(u)$ is invertible in $\mathcal{D}(R)$.

Added in proof. In a forthcoming paper by F. Mantese, A. Tonolo and the author we will generalize the results to the case of an $n$-tilting module.

\section{ACKNOWLEDGEMENTS}

I wish to thank Bernhard Keller for his help in proving a crucial step in the proof of Lemma 5.2 and Pedro Nicolás for suggesting the use of the powerful Proposition 1.3 in Gabriel and Zisman's book [14].

\section{REFERENCES}

[1] L. Angeleri Hügel, A. Tonolo, J. Trlifaj, Tilting preenvelopes and cotilting precovers, Algebr. Represent. Theory 4 (2001), 155-170. MR1834843 (2002e:16010)

[2] S. Bazzoni, Cotilting modules are pure-injective, Proc. Amer. Math. Soc. 131 (2003), 36653672. MR1998172 (2004f:16049)

[3] S. Bazzoni, D. Herbera, One dimensional tilting modules are of finite type, Algebr. Represent. Theory 11 (2008), no. 1, 43-61. MR 2369100(2009a:16010)

[4] M. Bökstedt and A. Neeman, Homotopy limits in triangulated categories, Compositio Math. 86 (1993), 209-234. MR 1214458 (94f:18008)

[5] S. Brenner, M. Butler, Generalizations of the Bernstein-Gelfand-Ponomarev reflection functors, Lecture Notes in Math., 832, Springer, 1980, 103-169. MR607151 (83e:16031)

[6] R. R. Colby, K. R. Fuller, Tilting, cotilting and serially tilted rings, Comm. Algebra 18(5) (1990), 1585-1615. MR.1059750 (91h:16011)

[7] R. Colpi, Tilting in Grothendieck categories, Forum Math. 11 (1999), 735-759. MR.1725595 (2000h:18018)

[8] R. Colpi and J. Trlifaj, Tilting modules and tilting torsion theories, J. Alg. 178 (1995), 614-634. MR1359905 (97e:16003)

[9] R. Colpi, G. D'Este, A. Tonolo, Quasi-tilting modules and counter equivalences, J. Alg. 191 (1997), 461-494. MR1448804 (98g:16003)

[10] R. Colpi, A. Tonolo, J. Trlifaj, Partial cotilting modules and the lattices induced by them, Comm. Algebra 25 (10) (1997), 3225-3237. MR.1465112 (98i:16003)

[11] A. Facchini, Divisible modules over integral domains, Ark. Mat. 26 (1988), no. 1, 67-85. MR.948281 (90a:13018)

[12] A. Facchini, A tilting module over commutative integral domains, Comm. Alg. 15(11) (1987), 2235-2250. MR912770 (89c:13028)

[13] L. Fuchs, On divisible modules over domains, Abelian groups and modules, Proc. of the Udine Conference, CISM Courses and Lectures, 287, Springer-Verlag, Wien-New York, 1984, 341-356. MR.789830 (86j:13012)

[14] P. Gabriel, M. Zisman, Calculus of fractions and homotopy theory, Ergebnisse der Mathematik und ihrer Grenzgebiete, 35, Springer-Verlag, New York, 1967. MR0210125 (35:1019)

[15] D. Happel, On the derived category of a finite-dimensional algebra, Comment. Math. Helv. 62 (1987), no. 3, 339-389. MR.910167 (89c:16029)

[16] D. Happel, C. Ringel, Tilted algebras, Trans. Amer. Math. Soc. 274 (1982), 399-443. MR675063 (84d:16027)

[17] D. Happel, I. Reiten, S. Smalø, Tilting in abelian categories and quasitilted algebras, Memoirs Amer. Math. Soc. 120, no. 575 (1996). MR1327209 (97j:16009) 
[18] B. Keller, Derived categories and their uses, Handbook of Algebra, Vol. 1, edited by M. Hazewinkel, North-Holland, Amsterdam, 1996. MR.1421815 (98h:18013)

[19] B. Keller, Derived categories and tilting, Handbook of Tilting Theory, LMS Lecture Note Series, 332, Cambridge University Press, Cambridge, 2007, 49-97. MR2384608(2009b:16029)

[20] N. Spaltenstein, Resolutions of unbounded complexes, Compositio Mathematica 65 (1988), 121-154. MR932640 (89m:18013)

[21] B. Stenström, Rings of quotients, Die Grundleheren der Math. Wissenschaften, 217, SpringerVerlag, New York-Heidelberg, 1975. MR0389953 (52:10782)

Dipartimento di Matematica Pura e Applicata, Università di Padova, Via Trieste 63, 35121 Padova, Italy

E-mail address: bazzoni@math.unipd.it 